\title{
Tuning the localized surface plasmon resonance of "core-shell Ag nanoparticles on dielectric substrates" to near-infrared window: applications to surface-enhanced Raman spectroscopy
}

\author{
SinA SALIMIAN, HADI SOOFI* \\ School of Engineering- Emerging Technologies, University of Tabriz, Tabriz 5166616471, Iran \\ ${ }^{*}$ Corresponding author: h.soofi@tabrizu.ac.ir
}

\begin{abstract}
In this article, plasmonic characteristics of $\mathrm{SiO}_{2}-\mathrm{Ag}$ and hollow core $\mathrm{Ag}$ nanoparticles placed on dielectric substrates are investigated and tuned to the NIR wavelength spectrum for biological applications. It is shown that by placing the core-shell Ag nanoparticles on a dielectric substrate and exciting the normal plasmon mode of the nanoparticle, it is possible to obtain strong plasmon resonances at wavelengths as long as $\lambda=700 \mathrm{~nm}$ which exhibits a red shift of more than $300 \mathrm{~nm}$ compared to the resonance of freestanding pure Ag nanoparticles at which normal plasmon resonance wavelength shows a sensitivity of approximately $100 \mathrm{~nm} / \mathrm{RIU}$ in respect to the substrate refractive index change. " $\mathrm{SiO}_{2}-\mathrm{Ag}$ and hollow core $\mathrm{Ag}$ nanoparticles on silicon" are optimized to exhibit a strong normal plasmon resonance at $\lambda=633 \mathrm{~nm}$ while preserving the plasmonic field enhancement intact. Finally, a three dimensional substrate for surface-enhanced Raman spectroscopy (SERS) is designed and numerically investigated. The substrate is composed of Si nanorod array decorated with the designed nanoparticles which exhibits superior characteristics such as a uniform and gapless field enhancement and an electromagnetic enhancement factor of more than $3 \times 10^{6}$, an order of magnitude higher than the enhancement factor for a similar structure decorated with Au nanoparticles.
\end{abstract}

Keywords: core-shell nanoparticles, plasmonics, Raman scattering.

\section{Introduction}

Excitation of conduction band electrons in metallic nanoparticles (NP) by an incident electromagnetic radiation, known as localized surface plasmon resonance (LSPR) is extensively studied in the past years [1] and it is shown that incoming photon can be effectively captured by the nanoparticle when the frequency of the photon is in resonance with the oscillation frequency of the electrons of the NP [2] . LSPR has found applications in surface enhanced sensing such as surface-enhanced Raman spectroscopy (SERS) [ $[\underline{3}, \underline{4}]$ and surface enhanced fluorescence [ $\underline{5}]$, photothermal therapy [ $[\underline{6}$, metamaterials [7], light emitting devices [ $\underline{8}$ ] and for light harvesting in solar cells []]. Among these applications, SERS is unique because of providing fingerprint spectra 
for various biological species. SERS applications include label-free detection of DNA $[10,11]$, intracellular monitoring [12], detection of cancer markers $[\underline{13}, 14]$ and many more. For biological applications such as SERS, plasmon resonance wavelength of NPs must be tuned to the so-called biological window $(\lambda=600-1350 \mathrm{~nm})[\underline{15}]$.

$\mathrm{Au}$ NPs exhibit strong LSPR in the visible to near IR wavelength range and hence are widely used to obtain electromagnetic enhancement for SERS applications [1], although further tuning of these NPs to observe a significant Raman signal or perform photothermal therapies is a crucial task. It is shown that Au nanostars have two distinct surface plasmon bands at visible and near infrared frequencies [16]. Gold nanocube dimers have been utilized for chemical sensing which show resonance at longer wavelengths than the Au nanospheres [17]. It is also shown that increasing the LSPR wavelength can be performed by altering the core-to-shell ratio at silica-Au core-shell and multilayer nanospheres [1] $]$. It is generally known that Ag NPs have higher absorption and scattering cross-sections than the Au NPs. However, the LSPR wavelength of these NPs is shorter than the wavelength of interest for the analysis of biological samples. For a single $\mathrm{Ag}$ nanosphere in air, $\lambda_{\mathrm{PR}}$ is less than $400 \mathrm{~nm}$ and for silver clusters another shoulder band appears at $500 \mathrm{~nm}$ [19]. Hence, SERS substrates utilizing Ag NPs have generally lower enhancement factors (EFs) than the gold counterparts. For instance, Ag NP decorated $\mathrm{Si} @ \mathrm{SiO}_{2}$ nanowire array exhibits an enhancement factor of $\mathrm{EF}=6 \times 10^{5}[\underline{20}]$, whereas a similar structure with Au NPs is demonstrated to have an EF of more than $10^{7}$ [21]. Application of Ag is mostly in bimetallic NPs or nanostructure assemblies such as Ag-Au normal and inverted core-shell nanospheres [22] and nanocubes [려] or complex structures such as Au nanostar-Ag NP satellite [24] and Au nanorod-Ag NP satellite [25] assemblies. Despite all the progress in the synthesis of complex NPs and NP assemblies, Au and Ag nanospheres are most favorable due to the simplicity of their fabrication process and their compatibility to various structures. Hence, tuning the plasmon characteristics of Ag NPs for biological applications is a crucial task.

Pioneering investigations on the contribution of dielectric substrates on the LSPR spectrum of Ag nanodiscs showed that LSPR wavelength experiences a red-shift by increasing the substrate refractive index [26]. At 2009, a study performed on Au/silica nanoshells and $\mathrm{Au}$ nanospheres showed that dielectric substrates lift the degeneracy of the dipole plasmon modes oriented parallel and normal to the substrate [27] at which the energy due to the normal orientation of plasmon mode is lower than the other. Fano resonances [28] induced by a dielectric substrate on the LSPR spectrum of a gold nanocube are investigated at [29]. Mathematical treatment of substrate-particle interaction is also performed at [30]. At a recent study, the optical properties of pure $\mathrm{Au}$ and $\mathrm{Ag}$ nanospheres above a dielectric substrate are investigated when there is a space between the NP and the substrate [31]. However, obtaining a strong plasmon resonance from tuned Ag NPs in the biological window has not been reported.

In this article, we investigate the optical properties of silica-silver and hollow core silver spherical core-shell NPs placed on Si substrates to tune the LSPR spectrum of 
these NPs for biological applications. The impact of the structural parameters of the NP such as the constituting materials, core and shell diameters as well as substrate refractive index and incoming light polarization on the plasmonic characteristics of the NP is investigated. Afterwards, $\mathrm{SiO}_{2}-\mathrm{Ag}$ and hollow core Ag spherical NPs are optimized to exhibit strong resonances at the popular SERS wavelength of $\lambda=633 \mathrm{~nm}$ when placed on silicon substrate and illuminated with a properly polarized light. Finally, a three dimensional SERS substrate composed of a silicon nanorod array functionalized with aforementioned NPs is designed and numerically investigated with the finite-difference time-domain (FDTD) method. In addition to the high electromagnetic enhancement factor (EMEF) of more than $3 \times 10^{6}$, which shows an improvement of at least an order of magnitude higher than the EMEF of the Si nanorod array decorated with $\mathrm{Au}$ NPs, the designed substrate provides uniform and gapless field enhancement which is evidenced through extensive numerical simulations.

\section{Theory and modeling}

Raman effect is a result of inelastic scattering between a photon and vibrational or rotational modes of a molecule. The power of the scattered Raman signal can be described by

$$
P_{\mathrm{S}}\left(v_{\mathrm{S}}\right)=N \sigma_{\mathrm{RS}} I\left(v_{\mathrm{L}}\right)
$$

where $N, \sigma_{\mathrm{RS}}$ and $I\left(v_{\mathrm{L}}\right)$ are the number of active scatters within the excitation spot, scattering cross section and intensity of an incoming beam at a frequency of $v_{\mathrm{L}}$ [32]; $v_{\mathrm{S}}$ is the frequency of the scattered Raman signal. Here, only Stokes shift is considered which means that the frequency of the Raman signal is less than the frequency of the incoming light which occurs where the incoming photon interacts with the molecule in its vibrational ground state.

In the presence of metallic nanostructures, this process can be enhanced which is known as SERS, provided that the scatters are located at the vicinity of these metallic nanostructures. In such cases, Eq. (1) is modified as

$$
P_{\mathrm{S}}\left(v_{\mathrm{S}}\right)=N \sigma_{\mathrm{SERS}}\left(\frac{E_{\mathrm{loc}}\left(v_{\mathrm{S}}\right)}{E_{0}} \frac{E_{\mathrm{loc}}\left(v_{\mathrm{L}}\right)}{E_{0}}\right)^{2} I\left(v_{\mathrm{L}}\right)
$$

where $E_{\text {loc }}$ and $E_{0}$ are the local field amplitude and the field amplitude of the laser beam; $\sigma_{\text {SERS }}$ is the scattering cross section which is different from $\sigma_{\mathrm{RS}}$ due to the fact that the presence of metallic nanostructures changes the environment of the molecule and hence modifies the scattering cross section. In Eq. (2), the second power is because the electromagnetic field intensity is proportional to the square of the field amplitude.

Enhancement of the Raman signal due to the increased scattering cross section known as the chemical enhancement is on the order of 100 [32] much less than the electromagnetic enhancement. As $v_{\mathrm{S}}$ and $v_{\mathrm{L}}$ are very close to each other, the electromagnetic enhancement factor is often written as 


$$
\mathrm{EMEF}=\frac{\left|E_{\mathrm{loc}}\right|^{4}}{\left|E_{0}\right|^{4}}
$$

which is known as the 4 th power or $|E|^{4}$ approximation.

Note that for practical situations and experiments, SERS enhancement factor is usually defined as

$$
\mathrm{EF}=\frac{I_{\mathrm{SERS}} / N_{\text {surf }}}{I_{\mathrm{RS}} / N_{\text {vol }}}
$$

where $I_{\mathrm{SERS}}$ and $I_{\mathrm{RS}}$ represent the Raman scattering intensities with and without the SERS effect, and the $N_{\text {surf }}$ and $N_{\text {vol }}$ are the corresponding numbers of molecules on the SERS-active surface and in the volume [33] .

For numerical simulations, EMEF is much more appropriate than the EF presented at Eq. (4) and is vastly utilized in the SERS context [34-36] which is also applied in this article. All numerical simulations throughout the article are performed with the FDTD method. For this analysis, perfectly matched layers (PML) are utilized along all directions, except for the 3D SERS substrate which will be discussed later. FDTD mesh side length is less than $2 \mathrm{~nm}$. However, for the plasmonic NPs, it is found that $2 \mathrm{~nm}$ is not enough as the electric field undergoes substantial variations near them. Hence the mesh size is decreased to $0.5 \mathrm{~nm}$ for them.

\section{Results and discussion}

At the first part of this section, the plasmonic characteristics of $\mathrm{SiO}_{2}$ and hollow core Ag NPs on dielectric substrates are analyzed and the sensitivity of the normal plasmon mode resonance on the dielectric refractive index is investigated to tune the NPs to the SERS applications. At the second part, a three dimensional SERS substrate composed of a silicon nanorod array functionalized with the designed NPs is presented and numerically investigated.

\subsection{Analysis of core-shell plasmonic nanospheres on dielectric substrates}

To obtain plasmon resonance at the biological window from core-shell Ag nanospheres, these nanoparticles are placed on a dielectric substrate with a refractive index of $n_{\mathrm{s}}$ and are illuminated with a light of arbitrary polarization as illustrated in Fig. 1. Particle and core diameter are denoted by $D_{\mathrm{p}}$ and $D_{\mathrm{c}}$ and their corresponding refractive indices are $n_{1}$ and $n_{2}$, respectively. $\mathrm{SiO}_{2}$ and air are utilized as the core dielectric materials because fabrication of both $\mathrm{SiO}_{2}-\mathrm{Ag}$ and hollow core $\mathrm{Ag}$ nanoparticles is previously reported $[\underline{37}, \underline{38}]$.

Absorption cross section versus wavelength for a pure Ag NP with a diameter of $D_{\mathrm{p}}=20 \mathrm{~nm}$ on a substrate with a refractive index of $n_{\mathrm{s}}=2.5$ and with a light polarization of $\theta=0,40^{\circ}$ and $60^{\circ}$ is illustrated in Fig. 2. Two peaks are visible in the cross section 

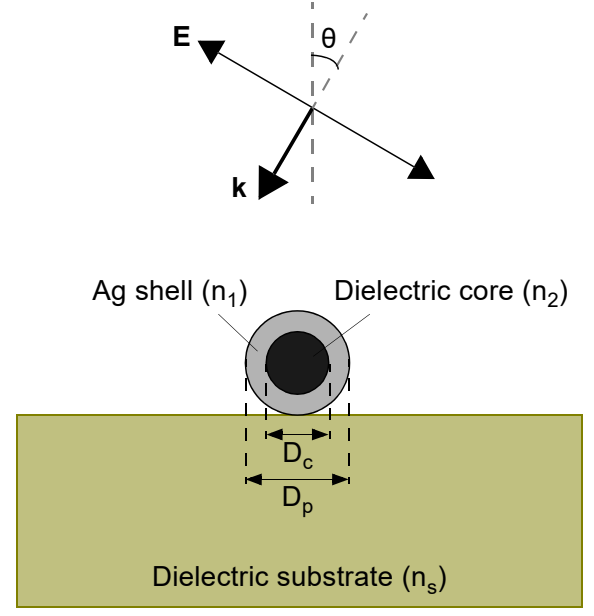

Fig. 1. Dielectric core-Ag shell NP placed on the surface of a dielectric substrate under illumination of a light source with polarization angle $\theta$.

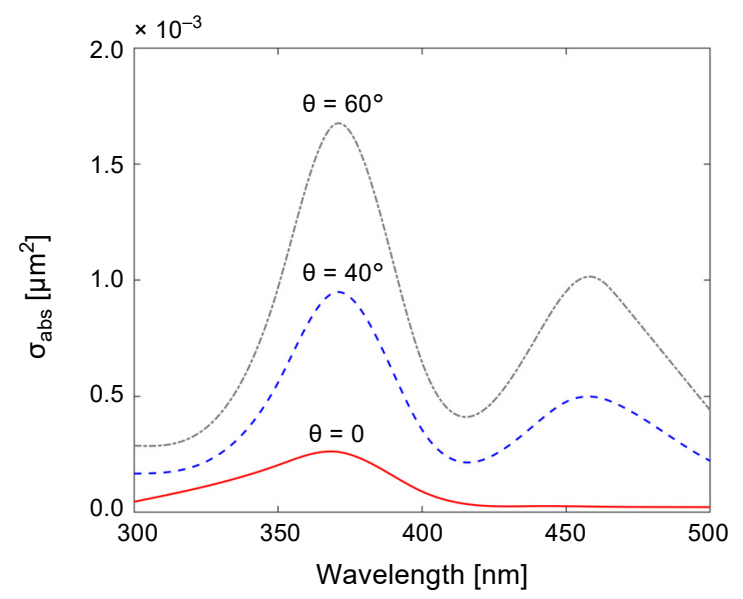

Fig. 2. Absorption cross section versus wavelength for a pure $\mathrm{Ag} \mathrm{NP}$ with $D_{\mathrm{p}}=20 \mathrm{~nm}$ placed on a substrate with $n_{\mathrm{s}}=2.5$ for $\theta=0,40^{\circ}$, and $60^{\circ}$. Two peaks at shorter and longer wavelengths in the spectrum correspond to longitudinal (LPR) and normal (NPR) plasmon resonances, respectively.

spectrum for both cases. First peak at $\lambda \approx 370 \mathrm{~nm}$ is due to the light polarization component parallel to the substrate surface which also polarizes the nanosphere in the same direction and hence, the substrate has no impact on this component which is called longitudinal plasmon resonance (LPR) here after. However, the other peak at longer wavelengths which is due to the plasmon excitation by the light polarized normal to the substrate surface has undergone a dramatic red shift. For the pure NP of Fig. 2, this resonance takes place at $\lambda_{\mathrm{NPR}} \approx 460 \mathrm{~nm}$ at which the subscript NPR stands for normal plasmon resonance. Red shift of NPR relative to LPR which is observed in various NP on substrate experiments $[\underline{26}, \underline{27}]$, is due to the fact that for the component of light 
polarized normal to the substrate surface, charges are localized near the substrate surface which results in a more pronounced interaction of them with the image charges of the "NP image" due to the presence of the dielectric surface. The interaction of NP with its image and the screening of electromagnetic fields is thoroughly discussed in [27]. Another interesting feature is that the wavelengths corresponding to LPR and NPR are $\theta$ independent as can be seen from Fig. 2. Hence, one can employ a light completely normal to the substrate surface to excite plasmons only at $\lambda_{\mathrm{NPR}}$.

Absorption cross section of the same pure Ag NP versus wavelength for various substrate refractive indices is illustrated in Fig. 3. For this analysis, light polarization angle is $\theta=20^{\circ}$ to excite NPR and LPR simultaneously. As expected, the shift of the LPR wavelength is quite negligible. However, NPR wavelength $\left(\lambda_{\mathrm{NPR}}\right)$ experiences a considerable red shift by increasing the substrate refractive index. For the case of $n_{\mathrm{s}}=2.5, \lambda_{\mathrm{NPR}} \approx 460 \mathrm{~nm}$ shifts to $\lambda_{\mathrm{NPR}} \approx 560 \mathrm{~nm}$ for $n_{\mathrm{s}}=3.5$. Hence the sensitivity of $\lambda_{\mathrm{NPR}}$ to refractive index change is approximately $100 \mathrm{~nm} / \mathrm{RIU}$ and is quite linear in the range of $n_{\mathrm{s}}=2.5-3.5$.

Figure 3 indicates that $\lambda_{\mathrm{NPR}} \approx 560 \mathrm{~nm}$ for $\mathrm{Ag} \mathrm{NP}$ on a substrate with $n_{\mathrm{s}}=3.5$. However, this value is still lower than the wavelength range of interest for biological applications. Here, to increase the NPR shift, dielectric core-Ag shell NPs are utilized and placed on a substrate with high refractive index such as silicon. By employing Mie theory calculations, it is shown that plasmon resonance wavelength of dielectric core-metal shell NPs shift toward longer wavelengths by increasing the relative core diameter [39]. Hence, it is expected that NPR also experiences such a red shift by increasing $D_{\mathrm{c}} / D_{\mathrm{p}}$. NPR wavelength and absorption efficiency for $\mathrm{SiO}_{2}$ core and hollow core Ag NPs on a silicon substrate versus $D_{\mathrm{c}} / D_{\mathrm{p}}$ are presented in Figs. $4 \mathbf{a}$ and $4 \mathbf{b}$, respectively. As expected, $\lambda_{\mathrm{NPR}}$ can be shifted to wavelengths as high as $\lambda=700 \mathrm{~nm}$ by increasing the relative core diameter. Hollow-core Ag NPs have higher absorption efficiencies and

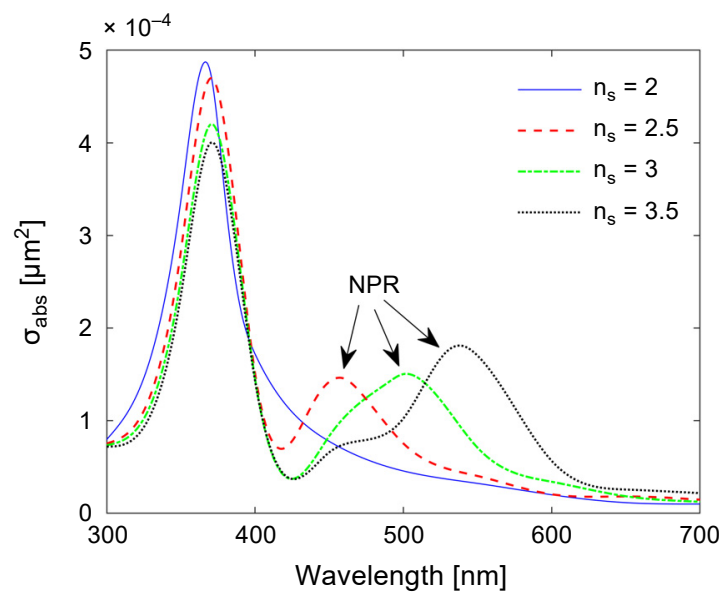

Fig. 3. Absorption cross section versus wavelength for a pure Ag NP placed on a substrate with refractive index $n_{\mathrm{s}}$ as indicated in the figure. 


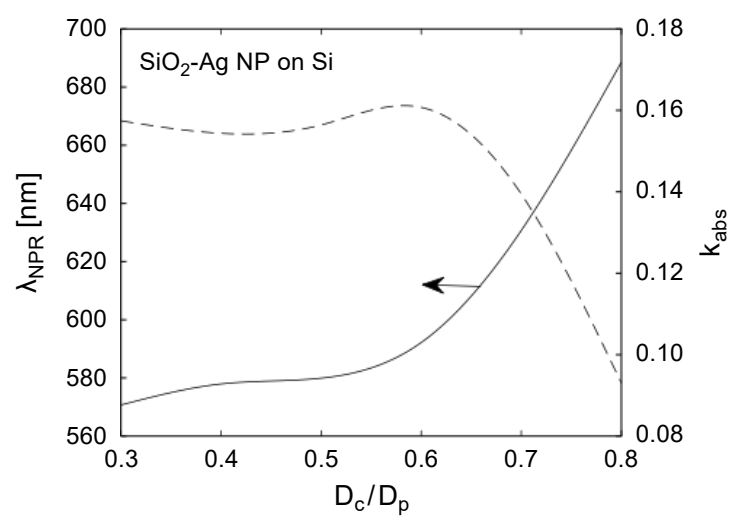

a

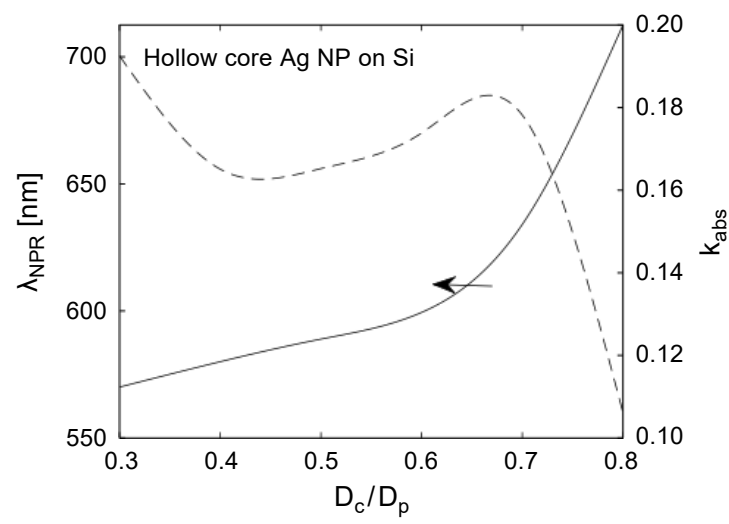

b

Fig. 4. Normal plasmon resonance and absorption efficiency versus relative core diameter $D_{\mathrm{c}} / D_{\mathrm{p}}$ for $\mathrm{SiO}_{2}$ core (a) and hollow core Ag NPs (b).

longer $\lambda_{\mathrm{NPR}}$ than the $\mathrm{SiO}_{2}$ core case. Another point that must be noted is that as $D_{\mathrm{c}} / D_{\mathrm{p}}$ increases beyond a threshold value, absorption efficiency drops rapidly due to the lower plasmonic effects for very thin metallic shells. This threshold value is 0.6 and 0.7 for $\mathrm{SiO}_{2}$ core and hollow core Ag NPs. Although this is not strict, $D_{\mathrm{c}} / D_{\mathrm{p}}$ can be increased beyond this value resulting in higher resonance wavelengths and slightly compromised plasmonic enhancements. As can be seen from Fig. $4, \lambda_{\mathrm{NPR}}$ can be tuned to approximately $\lambda=633 \mathrm{~nm}$ for " $\mathrm{SiO}_{2}$ core and hollow core Ag NPs on Si substrate" with $D_{\mathrm{p}}=20 \mathrm{~nm}$ and $D_{\mathrm{c}} / D_{\mathrm{p}}=0.7$.

\subsection{Design of the 3D SERS substrate}

In the previous section, it was shown that core-shell Ag NPs with $D_{\mathrm{p}}=20 \mathrm{~nm}$ and $D_{\mathrm{c}} / D_{\mathrm{p}}=0.7$ on a silicon substrate supports NPR wavelength at $\lambda_{\mathrm{NPR}}=633 \mathrm{~nm}$ when excited with a light polarized normal to the substrate surface. Based on these findings, a three dimensional SERS substrate is proposed which is schematically illustrated in Fig. 5. The structure incorporates silicon nanorods with a diameter of $D_{\mathrm{r}}$ and a height 


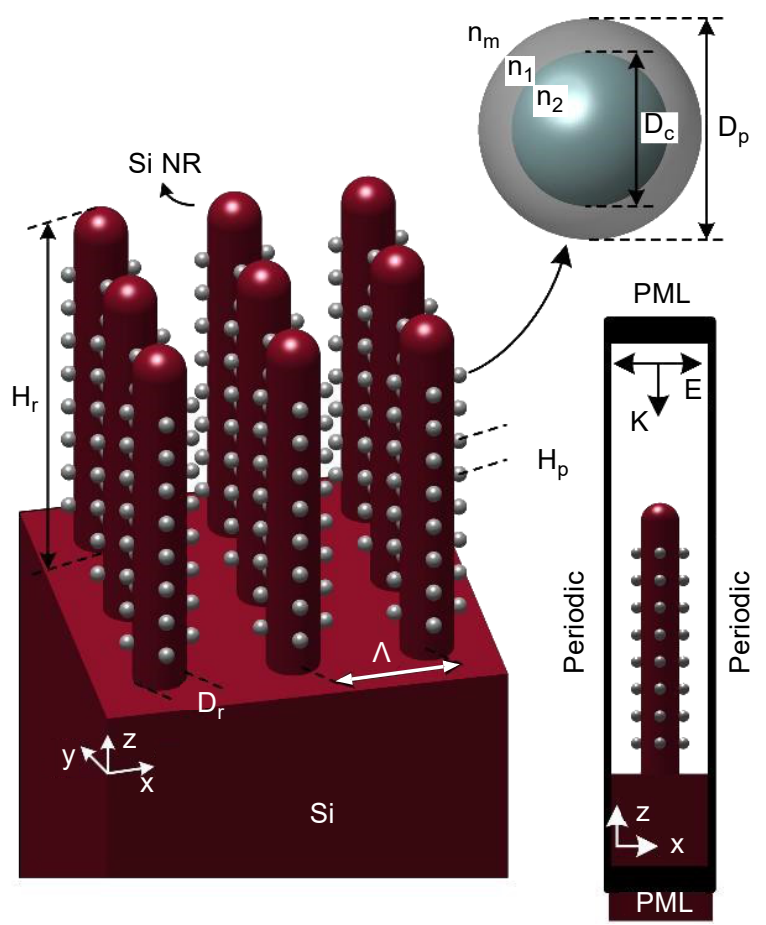

Fig. 5. A perspective view of the presented 3D SERS substrate composed of a Si nanorod array decorated with tuned Ag NPs. Similar structures with Au NPs are demonstrated previously [미].

of $H_{\mathrm{r}}$ arranged in a periodic square lattice with a pitch of $\Lambda$. Each nanorod is decorated with NPs designed at the previous section. Totally, 80 NPs (20 NPs in four columns) are utilized to decorate each silicon nanorod. Interparticle spacing of NPs in each column is $H_{\mathrm{p}}=60 \mathrm{~nm}$. Structural parameters for Si nanorod array are as follows: $D_{\mathrm{r}}=100 \mathrm{~nm}, H_{\mathrm{r}}=1.3 \mu \mathrm{m}, \Lambda=500 \mathrm{~nm}$. The pitch of $\Lambda=500 \mathrm{~nm}$ is chosen so that the gapless electrical field enhancement can be obtained, whereas being large enough to capture long chain molecules. 3D full vectorial FDTD simulations are performed to obtain the field enhancement characteristics of the presented SERS substrate. The simulation domain is also shown in Fig. 5. A plane wave source is polarized along $x$ direction and placed $400 \mathrm{~nm}$ above the structure. As can be seen, input light has a polarization normal to the nanorod axis and hence the requirement for successful excitation of NPR is fulfilled. The light wavelength is chosen to be $\lambda=633 \mathrm{~nm}$ matching the normal plasmon resonance wavelength of NPs designed in the previous section. It is worth noting that similar structures with pure Au NPs or untuned (pure) Ag NPs are previously demonstrated $[\underline{20}, \underline{21}]$ and the proposed structure is completely feasible. Here, it is shown that tuned Ag NPs in the previous section yield much higher enhancement factors and hence are much more suitable for SERS applications.

It was shown in previous articles that Ag NPs lead to lower EFs [20] than Au NPs [21] for a similar structure due to the mismatch between the plasmon resonance wavelength 


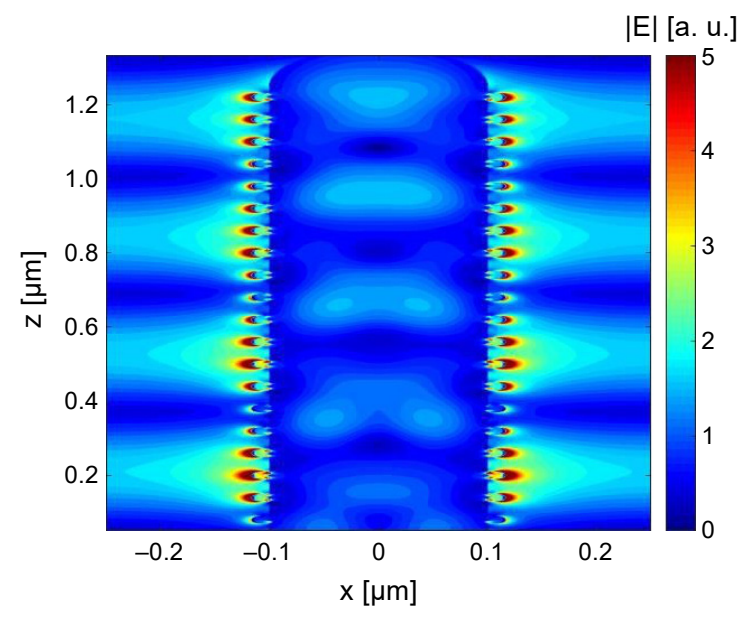

Fig. 6. Electric field magnitude at a unit cell of the structure at a wavelength of $\lambda=633 \mathrm{~nm}$.

of untuned Ag NPs with the laser light of $\lambda=633 \mathrm{~nm}$. In this article, a systematic approach is presented to tune the LSPR resonance frequency of silver NPs to the desired wavelength. Hence, it is expected to obtain higher field enhancement than the Au nanoparticle decorated case. For comparison purposes, the structure presented in Fig. 5 is numerically simulated with pure Au NPs either. These NPs are considered to be nanospheres with the same diameter of $D_{\mathrm{p}}=20 \mathrm{~nm}$ which have strong LSPR at $\lambda=633 \mathrm{~nm}$ [21]. Electric field amplitude along the structure cross-section for a unit cell of the SERS substrate is presented in Fig. 6. For this figure, decorator NPs are hollow core Ag nanospheres with $D_{\mathrm{p}}=20 \mathrm{~nm}$ and $D_{\mathrm{c}} / D_{\mathrm{p}}=0.7$. As can be seen, the normalized electric field amplitude far from the NR reaches a value of approximately 2 , hence providing the gapless property. Although the color bar is fixed to a maximum value of 5 , however, the maximum electric field amplitude is actually higher reaching to almost 12 times the input source amplitude near the NP surfaces.

To better illustrate the advantage of the presented core-shell Ag NPs compared to conventional Au NPS, the number of hotspots in the structure is counted. The electrical field amplitude in a $x z$ plane passing through the axis of the nanorod is recorded for a unit cell of the structure. Each FDTD mesh at which the normalized electrical field magnitude is higher than a threshold value $\left|E_{\mathrm{th}}\right|$ is considered as a hotspot. The number of hotspots versus the threshold value is illustrated in Fig. 7 at which $\left|E_{0}\right|$ is the source of electric field amplitude. As can be seen, for Si nanorods decorated with Au NPs, less than 20 meshes reach an electric field amplitude 10 times of the source amplitude. However, this value is more than 100 for $\mathrm{SiO}_{2} @ \mathrm{Ag}$ and hollow core Ag NPs which proves that tuning of these NPs to $\lambda=633 \mathrm{~nm}$ has been performed successfully.

As discussed in Section 2, an electromagnetic enhancement factor (EMEF) can be effectively utilized to compare the field enhancement of SERS substrates. Here, this value is employed to compare the designed substrates utilizing core-shell AgNPs with the substrate decorated with pure Au NPs. By utilizing Eq. (4) and considering each mesh 


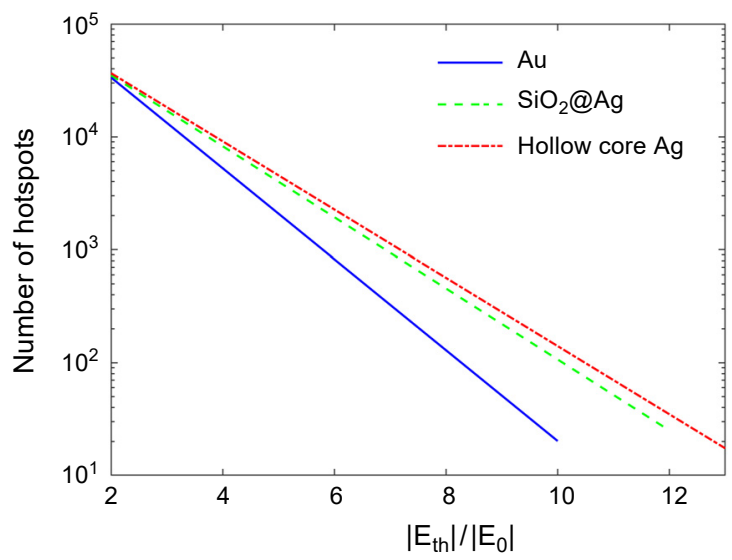

Fig. 7. Number of hotspots in an $x z$ plane passing through the axis of a Si nanorod at each unit cell of the structure for three decorators $\left(\mathrm{Au}, \mathrm{SiO}_{2} @ \mathrm{Ag}\right.$ and hollow core Ag NPs).

with a normalized electric field amplitude of at least 10 as a hotspot, the EMEF of the Si nanorod array decorated with pure $\mathrm{Au}, \mathrm{SiO}_{2}$ core and hollow core Ag NPs are calculated to be $1.26 \times 10^{5}, 1.83 \times 10^{6}$ and $3.04 \times 10^{6}$, respectively. The results indicate that the EMEF is enhanced more than an order of magnitude in the designed substrates compared to the same substrate covered with Au nanoparticles.

\section{Conclusion}

In this article, plasmonic characteristics of $\mathrm{SiO}_{2}-\mathrm{Ag}$ and hollow core $\mathrm{Ag}$ NPs placed on dielectric substrates are investigated and it is shown that normal plasmon resonance wavelength of the "NP on a dielectric substrate" which is excited by a light polarized normal to the substrate surface, exhibits a strong sensitivity to the substrate refractive index on the order of $100 \mathrm{~nm} / \mathrm{RIU}$ which can be utilized to tune the resonance of these NPs to the biological window. $\mathrm{SiO}_{2}-\mathrm{Ag}$ and hollow core $\mathrm{Ag}$ NPs are tuned to exhibit a strong normal plasmon mode resonance at a wavelength of $\lambda=633 \mathrm{~nm}$ (a popular wavelength in SERS applications) when placed on a Si substrate. Geometrical parameters of the nanoparticles are a relative core diameter of $D_{\mathrm{c}} / D_{\mathrm{p}}=0.7$ and a particle size of $D_{\mathrm{p}}=20 \mathrm{~nm}$. Finally, a three dimensional SERS substrate composed of a Si nanorod array decorated with the designed NPs is presented and numerically investigated. The SERS substrate shows superior characteristics such as uniform and gapless field enhancement and an EMEF of larger than $3 \times 10^{6}$, which is more than an order of magnitude higher than similar structures with Au NPs or untuned Ag NPs. The systematic approach taken in this article, makes it possible to extend the nanoparticle design strategy to various nanoparticle shapes and substrates.

Acknowledgments - This research did not receive any specific grant from funding agencies in the public, commercial, or not-for-profit sectors. 


\section{References}

[1] Amendola V., Pilot R., Frasconi M., Marago O.M., Iati M.A., Surface plasmon resonance in gold nanoparticles: a review, Journal of Physics: Condensed Matter 29(20), 2017, article 203002, DOI: 10.1088/1361-648X/aa60f3.

[2] Biagioni P., Huang J.S., Hecht B., Nanoantennas for visible and infrared radiation, Reports on Progress in Physics 75(2), 2012, article 024402, DOI: 10.1088/0034-4885/75/2/024402.

[3] Le Ru E.C. Etchegoin P., Principles of Surface-Enhanced Raman Spectroscopy: and Related Plasmonic Effects, Elsevier Science, 2009, DOI: 10.1016/B978-0-444-52779-0.X0001-3.

[4] Haes A.J., Haynes C.L., McFarland A.D., Schatz G.C., Van Duyne R.P., Zou S., Plasmonic materials for surface-enhanced sensing and spectroscopy, MRS Bulletin 30(5), 2005, pp. 368-375, DOI: $10.1557 / \mathrm{mrs} 2005.100$.

[5] KÜHn S., HǍkanson U., Rogobete L., SANDOghdar V., Enhancement of single-molecule fluorescence using a gold nanoparticle as an optical nanoantenna, Physical Review Letters 97(1), 2006, article 017402, DOI: 10.1103/PhysRevLett.97.017402.

[6] Wang Y., Black K.C., Luehmann H., Li W., Zhang Y., Cai X., Wan D., Liu S.Y., Li M., Kim P., Li Z.Y., Wang L.V., LiU Y., XIA Y., Comparison study of gold nanohexapods, nanorods, and nanocages for photothermal cancer treatment, ACS Nano 7(3), 2013, pp. 2068-2077, DOI: $10.1021 /$ nn304332s.

[7] Xu W., Xie L., Zhu J., Xu X., Ye Z., Wang C., Ma Y., Ying Y., Gold nanoparticle-based terahertz metamaterial sensors: mechanisms and applications, ACS Photonics 3(12), 2016, pp. 2308-2314, DOI: $10.1021 /$ acsphotonics.6b00463.

[8] Lozano G., Rodriguez S.R.K., Verschuuren M.A., Rivas J.G., Metallic nanostructures for efficient LED lighting, Light: Science and Applications 5(6), 2016, article e16080, DOI: 10.1038/1sa.2016.80.

[9] Atwater H.A. Polman A., Plasmonics for improved photovoltaic devices, Nature Materials 9(3), 2010, pp. 205-213, DOI: $10.1038 /$ nmat2629.

[10] Su J., Wang D., Nörbel L., Shen J., Zhao Z., Dou Y., Peng T., Shi J., Mathur S., Fan C., Song S., Multicolor gold-silver nano-mushrooms as ready-to-use SERS probes for ultrasensitive and multiplex DNA/miRNA detection, Analytical Chemistry 89(4), 2017, pp. 2017-2531, DOI: $10.1021 /$ acs.analchem.6b04729.

[11] Guselnikova O., Postnikov P., Pershina A., Svorcik V., Lyutakov O., Express and portable label -free DNA detection and recognition with SERS platform based on functional Au grating, Applied Surface Science 470, 2019, pp. 219-227, DOI: 10.1016/j.apsusc.2018.11.092.

[12] Shen Y., Liang L., Zhang S., Huang D., Zhang J., Xu S., Liang C., Xu W., Organelle-targeting surface-enhanced Raman scattering (SERS) nanosensors for subcellular pH sensing, Nanoscale 10, 2018, pp. 1622-1630, DOI: 10.1039/C7NR08636A.

[13] Wang Z., Zong S., Wang Y., Li N., Li L., Lu J., Wang Z., Chen B., Cui Y., Screening and multiple detection of cancer exosomes using an SERS-based method, Nanoscale 10, 2018, pp. 9053-9062, DOI: 10.1039/C7NR09162A.

[14] Ravanshad R., Zadeh A.K., Amani A.M., Mousavi S.M., Hashemi S.A., Dashtaki A.S., Mirzaei E., ZARE B., Application of nanoparticles in cancer detection by Raman scattering based techniques, Nano Reviews \& Experiments 9(1), 2018, article 1373551, DOI: 10.1080/20022727.2017.1373551.

[15] Smith A.M., Mancini M.C., Nie S., Bioimaging: second window for in vivo imaging, Nature Nanotechnology 4(11), 2009, pp. 710-711, DOI: 10.1038/nnano.2009.326.

[16] Tian F., Conde J., Bao C., Chen Y., Curtin J., Cui D., Gold nanostars for efficient in vitro and in vivo real-time SERS detection and drug delivery via plasmonic-tunable Raman/FTIR imaging, Biomaterials 106, 2016, pp. 87-97, DOI: $\underline{10.1016 / \text { j.biomaterials.2016.08.014. }}$

[17] Omar R., Naciri A.E., Jradi S., Battie Y., Toufaily J., Mortada H., Akil S., One-step synthesis of a monolayer of monodisperse gold nanocubes for SERS substrates, Journal of Materials Chemistry C 5(41), 2017, pp. 10813-10821, DOI: 10.1039/C7TC03069J. 
[18] Gao Y., Li Y., Wang Y., Chen Y., Gu J., Zhao W., Ding J., Shi J., Controlled synthesis of multilayered gold nanoshells for enhanced photothermal therapy and SERS detection, Small 11(1), 2015, pp. 77 -83, DOI: $10.1002 / \mathrm{smll} .201402149$.

[19] Cholula-Díaz J.L., Lomelí-Marroquín D., Pramanick B., Nieto-Argüello A., Cantú-Castillo L.A., HwANG H., Synthesis of colloidal silver nanoparticle clusters and their application in ascorbic acid detection by SERS, Colloids and Surfaces B: Biointerfaces 163, 2018, pp. 329-335, DOI: $10.1016 /$ j.colsurfb.2017.12.051.

[20] Liu X.Y., Huang J.A., YAng B., Zhang X.J., Zhu Y.Y., Highly reproducible SERS substrate based on polarization-free Ag nanoparticles decorated $\mathrm{SiO}_{2} /$ Si core-shell nanowires array, AIP Advances 5(5), 2015, article 057159, DOI: 10.1063/1.4921943.

[21] Lin D., Wu Z., Li S., Zhao W., Ma C., Wang J., Jiang Z., Zhong Z., Zheng Y., Yang X., Large-area Au-nanoparticle-functionalized Si nanorod arrays for spatially uniform surface-enhanced Raman spectroscopy, ACS Nano 11(2), 2017, pp. 1478-1487, DOI: 10.1021/acsnano.6b06778.

[22] Mohan S., Chandrasekar A., Subramanian B., Surface enhanced Raman scattering studies of silver-gold normal and inverted core-shell nanostructures on their efficiency of detecting molecules, Procedia Engineering 92, 2014, pp. 19-25, DOI: 10.1016/j.proeng.2013.10.005.

[23] Guo P., Sikdar D., Huang X., Si K.J., Xiong W., Gong S., Yap L.W., Premaratne M., Cheng W., Plasmonic core-shell nanoparticles for SERS detection of the pesticide thiram: size- and shape-dependent Raman enhancement, Nanoscale 7, 2015, pp. 2862-2868, DOI: 10.1039/C4NR06429A.

[24] Li A., Tang L., Song D., Song S., Ma W., Xu L., Kuang H., Wu X., Liu L., Chen X., Xu C., A SERS -active sensor based on heterogeneous gold nanostar core-silver nanoparticle satellite assemblies for ultrasensitive detection of aflatoxinB1, Nanoscale 8(4), 2016, pp. 1873-1878 DOI: 10.1039/ C5NR08372A.

[25] Xu L., Kuang H., Xu C., Ma W., Wang L., Kotov N.A., Regiospecific plasmonic assemblies for in situ Raman spectroscopy in live cells, Journal of the American Chemical Society 134(3), 2012, pp. 1699-1709, DOI: $10.1021 / \mathrm{ja} 2088713$.

[26] Malinsky M.D., Kelly K.L., Schatz G.C., Van Duyne R.P., Nanosphere lithography: effect of substrate on the localized surface plasmon resonance spectrum of silver nanoparticles, The Journal of Physical Chemistry B 105(12), 2001, pp. 2343-2350, DOI: 10.1021/jp002906x.

[27] Knight M.W., Wu Y., Lassiter J.B., Nordlander P., Halas N.J., Substrates matter: influence of an adjacent dielectric on an individual plasmonic nanoparticle, Nano Letters 9(5), 2009, pp. 2188 -2192 , DOI: $10.1021 / \mathrm{n} 1900945 \mathrm{q}$.

[28] Luk'yanchuk B., Zheludev N.I., Maier S.A., Halas N.J., Nordlander P., Giessen H., Chong C.T., The Fano resonance in plasmonic nanostructures and metamaterials, Nature Materials 9(9), 2010, pp. 707-715, DOI: 10.1038/nmat2810.

[29] Zhang S., Bao K., Halas N.J., Xu H., Nordlander P., Substrate-induced Fano resonances of a plasmonic nanocube: a route to increased-sensitivity localized surface plasmon resonance sensors revealed, Nano Letters 11(4), 2011, pp. 1657-1663, DOI: 10.1021/n1200135r.

[30] Vernon K.C., Funston A.M., Novo C., Gómez D.E., Mulvaney P., Davis T.J., Influence of particle -substrate interaction on localized plasmon resonances, Nano Letters 10(6), 2010, pp. 2080-2086, DOI: $10.1021 / \mathrm{nl} 100423 \mathrm{z}$.

[31] Lermé J., Bonnet C., Broyer M., Cottancin E., Manchon D., Pellarin M., Optical properties of a particle above a dielectric interface: cross sections, benchmark calculations, and analysis of the intrinsic substrate effects, The Journal of Physical Chemistry C 117(12), 2013, pp. 6383-6398, DOI: $10.1021 / \mathrm{jp} 3121963$.

[32] Maier S.A., Plasmonics: Fundamentals and Applications, Springer, New York, NY, 2007, DOI: 10.1007/0-387-37825-1.

[33] Kalachyova Y., Mares D., Jerabek V., Zaruba K., Ulbrich P., Lapcak L., Svorcik V., Lyutakov O., The effect of silver grating and nanoparticles grafting for LSP-SPP coupling and SERS response intensification, The Journal of Physical Chemistry C 120(19), 2016, pp. 10569-10577, DOI: 10.1021/ acs.jpcc.6b01587. 
[34] Elsayed M.Y., Gouda A.M., Ismail Y., Swillam M.A., Silicon-based SERS substrates fabricated by electroless etching, Journal of Lightwave Technology 35(14), 2017, pp. 3075-3081.

[35] Wei W., Du Y., Zhang L., Yang Y., Gao Y., Improving SERS hot spots for on-site pesticide detection by combining silver nanoparticles with nanowires, Journal of Materials Chemistry C 6(32), 2018, pp. 8793-8803, DOI: 10.1039/C8TC01741G.

[36] Wei Y., Pei H., Sun D., Duan S., Tian G., Numerical investigations on the electromagnetic enhancement effect to tip-enhanced Raman scattering and fluorescence processes, Journal of Physics: Condensed Matter 31(23), 2019, article 235301, DOI: 10.1088/1361-648X/ab0b9d.

[37] Semenova A.A., Semenov A.P., Gudilina E.A., Sinyukova G.T., Brazhe N.A., Maksimov G.V., GoodiLIN E.A., Nanostructured silver materials for noninvasive medical diagnostics by surface-enhanced Raman spectroscopy, Mendeleev Communications 26(3), 2016, pp. 177-186, DOI: 10.1016 / j.mencom.2016.04.001.

[38] Li C., Liu H., Cui P., YE F., YANG J., Insight into the formation of hollow silver nanoparticles using a facile hydrothermal strategy, Particuology 24, 2016, pp. 197-202, DOI: 10.1016/j.partic.2015.08.003.

[39] Xue X., Sukhotskiy V., Furlani E.P., Optimization of optical absorption of colloids of SiO $@$ @Au and $\mathrm{Fe}_{3} \mathrm{O}_{4} @$ Au nanoparticles with constraints, Scientific Reports 6, 2016, article 35911, DOI: 10.1038/srep35911. 